\title{
Estilo de vida y su relación con el exceso de peso, en los médicos residentes de un hospital nacional
}

Association of lifestyle and overweight in medical residents at a national

hospital

\author{
Jhon Cuba' ${ }^{1}$, Tati Ramírez ${ }^{1}$, Bárbara Olivares ${ }^{1}$, Ivonne Bernui ${ }^{2}$, Enriqueta Estrada ${ }^{2}$ \\ 'Internos EAP de Nutrición, Facultad de Medicina, Universidad Nacional Mayor de San Marcos. \\ ${ }^{2}$ Docentes, Facultad de Medicina, UNMSM.
}

\begin{abstract}
Resumen
Introducción: El exceso de peso en los últimos años se ha visto incrementado a nivel mundial. El médico, siendo parte de la sociedad, es la primera imagen en la atención en salud y debería tener condiciones adecuadas de salud. Objetivos: Determinar los estilos de vida y su relación con el exceso de peso en el personal médico. Diseño: Estudio correlacional, observacional, prospectivo, transversal. Institución: Hospital Nacional Daniel Alcides Carrión, Lima, Perú. Participantes: Médicos residentes. Intervenciones: Se estudió una muestra de 34 médicos residentes comprendidos entre 25 y 60 años. Se tomó peso y talla, para determinar el índice de masa corporal, y se aplicó una encuesta sobre estilos de vida. Principales medidas de resultados: Estilos de vida y su relación con el exceso de peso. Resultados: De los 34 residentes, $88 \%$ fueron del sexo masculino y $12 \%$ del femenino; la edad promedio fue $31,5 \pm 7,5$. El $53 \%$ de la población presentó sobrepeso y $21 \%$ obesidad. En relación al estilo de vida, los residentes empleaban 9 minutos para el desayuno, 26 minutos para el almuerzo y 27 para la cena; $88 \%$ y $82 \%$, respectivamente, consumían frutas y verduras por debajo de lo recomendado; $71 \%$ consumía alimentos entre comidas, de los cuales $69 \%$ presentaba exceso de peso. Un $46 \%$ de los que presentaba exceso de peso preferia alimentos altos en condimentos y $65 \%$ de ellos ingeria alimentos en un tiempo menor de 2 horas antes de dormir. El $91 \%$ continuaba trabajando posteriormente a sus guardias nocturnas, $74 \%$ no realizaba actividad física programada y $65 \%$ se percibia estresado. Conclusiones: Existió una relación de $74 \%$ de exceso de peso en los médicos residentes del HNDAC; sin embargo, no se encontró correlación significativa con el estilo de vida.
\end{abstract}

Palabras clave: Estilo de vida, exceso de peso.

\section{Abstract}

Background: In recent years weight excess has increased worldly. The physician is part of society and the first image in health care and should be adequately healthy. Objectives: To correlate lifestyle and overweight in medical residents. Design: Correlation, observational, prospective, transversal study. Setting: Hospital Nacional Daniel Alcides Carrion, Lima, Peru. Participants: Medical residents. Interventions: A sample of 34 medical residents 25 through 60 year-old was studied. Weight and height were obtained for body mass index and a lifestyle survey was applied. Main outcome measures: Lifestyle relation to weigh excess. Results: From 34 residents $88 \%$ were male and $12 \%$ female; average age was $31,5 \pm 7,5 ; 53 \%$ presented overweight and $21 \%$ obesity. In relation to lifestyle subjects spent 9 minutes for breakfast, 26 minutes for lunch and 27 for dinner; $88 \%$ and $82 \%$ respectively consumed fruits and vegetables below recommendations; $71 \%$ had snacks between meals and $69 \%$ of them were overweight; $46 \%$ of those overweight preferred high seasoned foods and $65 \%$ ate foods less than two hours before sleeping; $91 \%$ continued working after night calls; $74 \%$ did not have programmed physical activity and $65 \%$ considered himself stressed. Conclusions: There existed $74 \%$ weight excess in this hospital medical residents, but it did not correlate with lifestyle.

Key words: Lifestyle, weight excess.

An Fac med. 2011;72(3):205-10 


\section{INTRODUCCIÓN}

El sobrepeso y la obesidad han incrementado a nivel mundial, incluyendo América Latina, así como las enfermedades asociadas a estos excesos, como la diabetes mellitus II, cardiopatía coronaria, hipertensión arterial y cáncer. La obesidad influye en la calidad de vida y la capacidad laboral. Thompson y col. refieren que la obesidad disminuye la capacidad de las personas para realizar sus labores ${ }^{(1)}$.

La obesidad es de etiología multicausal, siendo sus principales factores biológico, social y psicológico. Entre ellos podemos mencionar como los más resaltantes a los cambios de hábitos alimentarios -consumo excesivo de alimentos con contenido alto de energía, abuso de condimentos-, consumo de alcohol y tabaco y sedentarismo, que va en aumento en las ciudades urbanizadas, principalmente por la facilidad del transporte y la tecnología (2).

Arenas, en México, el año 2004, señala que cualquier profesión u oficio conduce a que se conforme un hábito propio de la profesión y que dicho hábito está constituido por un conjunto de técnicas, referencias, creencias que se constituyen en estructuras y esquemas mentales; estas llevan a que las personas tengan determinadas prácticas. El conocimiento técnico adquirido durante la formación académica le confiere a la profesión médica una característica singular: derecho a controlar su propio trabajo, que se refleja, en parte, en la autonomía para establecer el diagnóstico y el tratamiento médico ${ }^{(3)}$. El autor indica que este hecho puede conducir a que los médicos tomen conciencia de la vulnerabilidad de los seres humanos y de ellos mismos; esta vulnerabilidad es un recordatorio persistente de nuestra mortalidad. Sin embargo, pareciera que este suceso puede conducir también a lo contrario, al supuesto de que por tener los conocimientos aproximados o acertados acerca de la enfermedad y la muerte se tiene la posibilidad de manejarse con cierta dosis de invulnerabilidad o, bien, como lo menciona Berger, arrogancia médica, lo que lleva a que los médicos incurran en prácticas nocivas para sí mismos y que estas tengan significados y explicaciones a partir del cuerpo de conocimientos técnicos que poseen ${ }^{(3)}$.

Existen otras consideraciones que, añadidas a las ya mencionadas, pueden influir en la existencia de sobrepeso u obesidad, como son el tabaquismo y las características socioeconómicas; es decir, a mayor ingreso económico mayor presencia del sobrepeso u obesidad; también, se encuentra una relación directamente proporcional con el nivel de urbanización y la presencia de sobrepeso u obesidad en conjunto con mayores ingresos ${ }^{(1)}$.

Según la Encuesta Nacional de Niveles de Vida (ENNIV) - Perú, en el año 2000, se informó que el porcentaje de hombres que consumen tabaco y presentan sobrepeso u obesidad es $57,1 \%$ y, los que no lo hacen, $48 \%{ }^{(4)}$. Según la Organización Panamericana de la Salud, en el Perú, para el año 2005, 20\% de las mujeres y $12 \%$ de los varones entre 15 y 49 años de edad presentaban obesidad ${ }^{(5)}$. De acuerdo a la Asociación para el estudio de la Obesidad y Ateroesclerosis, en Lima metropolitana, la prevalencia para el año 2005 fue $40,4 \%$ para sobrepeso y $18,7 \%$ para obesidad ${ }^{(6)}$. El personal médico, siendo parte de la sociedad en su conjunto, no se encuentra exento de la problemática del sobrepeso u obesidad, ya que también puede presentar inactividad física, ingesta de alcohol, ingesta de alimentos ricos en energía, entre otros. Ello es de vital importancia, por ser personal que se encuentra en contacto directo con pacientes y su salud no puede verse deteriorada, más aún, por ser imagen y ejemplo para con los pacientes.

Por lo antes mencionado, se consideró investigar respecto a los estilos de vida y su relación con la prevalencia del exceso de peso en el personal médico.

\section{MÉTODOS}

El presente estudio es de tipo observacional, correlacional, prospectivo y transversal. La población de estudio fue la de médicos residentes del Hospital Nacional Daniel A. Carrión, Callao, Perú. El método de muestreo fue no probabilístico, por conveniencia; la muestra fue de 34 médicos residentes de ambos sexos. Los criterios de inclusión fueron: laborar más de 6 meses en la institución, rango de edad de 25 a 60 años y firmar el consentimiento informado. Se excluyó de la muestra aquellos residentes que presentaron alguna patología, que se encontraran gestando o en etapa de lactancia.

La toma del peso se obtuvo con ropa ligera y sin zapatos, con balanza de baño con capacidad de $150 \mathrm{~kg}$ y con precisión de $100 \mathrm{~g}$, y la talla mediante un tallímetro certificado por el Centro Nacional de Alimentación y Nutrición (CENAN), con precisión de $1 \mathrm{~mm}$. En base a los datos antropométricos, se determinó el índice de masa corporal, usando la clasificación de acuerdo a los parámetros de la Organización Mundial de la Salud y del Ministerio de Salud, indicando como normales de 18,5 $\mathrm{kg} / \mathrm{m}^{2}$ a $24,5 \mathrm{~kg} / \mathrm{m}^{2}$, sobrepeso de 25,0 $\mathrm{kg} / \mathrm{m}^{2}$ a $29,9 \mathrm{~kg} / \mathrm{m}^{2}$ y obesidad mayor o igual de $30 \mathrm{~kg} / \mathrm{m}^{2}$.

Tabla 1. Situación nutricional según rango de edad de los médicos residentes.

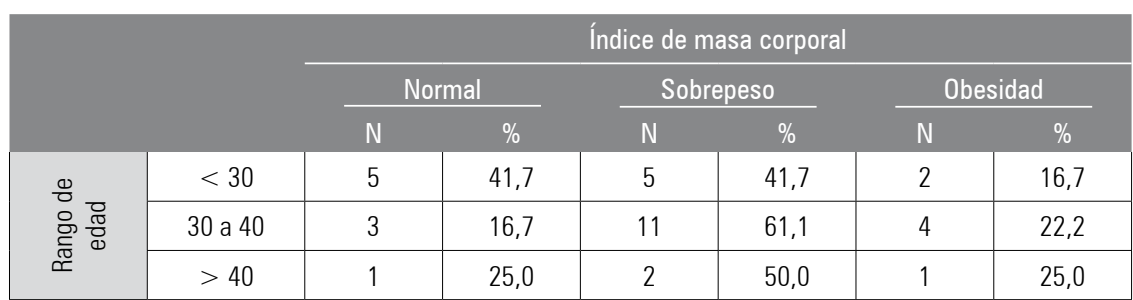


Para determinar el estilo de vida, se aplicó una encuesta, la que enmarca tres categorías: alimentación, actividad física y hábitos nocivos. La categoría alimentación se basó en 9 preguntas sobre frecuencia de consumo de comidas en casa, frecuencia de consumo de frutas y verduras (se consideró como cuántas veces al día se consumió, refiriéndose a raciones), consumo de pan, consumo de alimentos entre comidas, nivel de condimentación, tiempo dedicado a las comidas principales, alimentacióndescanso, alimentación-trabajo. La actividad física se basó en 5 preguntas sobre el tipo de actividad, frecuencia y tiempo del mismo; también se incluyó el descanso luego del trabajo. Y para los hábitos nocivos, se consideró el consumo de alcohol y el fumar.

La información obtenida fue procesada mediante el programa estadístico SPSS versión 13,0. La relación entre las variables fue evaluada con la prueba chi-cuadrado, valor de Yates (por tratarse de una muestra menor de 40). Para la elaboración de la base de datos y los gráficos, se utilizó el programa Microsoft Excel 2007.

\section{RESULTADOS}

La población estudiada fue predominantemente de varones (34 residentes, $88 \%)$. Según el índice de masa corporal, 16 (54\%) varones tenían sobrepeso y $7(23 \%)$, obesidad. Para el sexo femenino, 2 (50\%) tuvieron sobrepeso y 2 peso normal; 7 (23\%) varones presentaban obesidad, pero ninguna de las mujeres la tenía.

La mayor parte de la muestra estuvo comprendida entre los 30 y 40 años de edad y la frecuencia de sobrepeso y obesidad también fue mayor en dicho grupo, seguido por el grupo de edad mayor de 40 años (tabla 1).

La frecuencia con la que se consumía las diferentes comidas principales (desayuno, almuerzo, cena), podemos observar (tabla 2) que el almuerzo fue el tiempo de comida que se realizó con menor frecuencia en casa (3 veces/se-

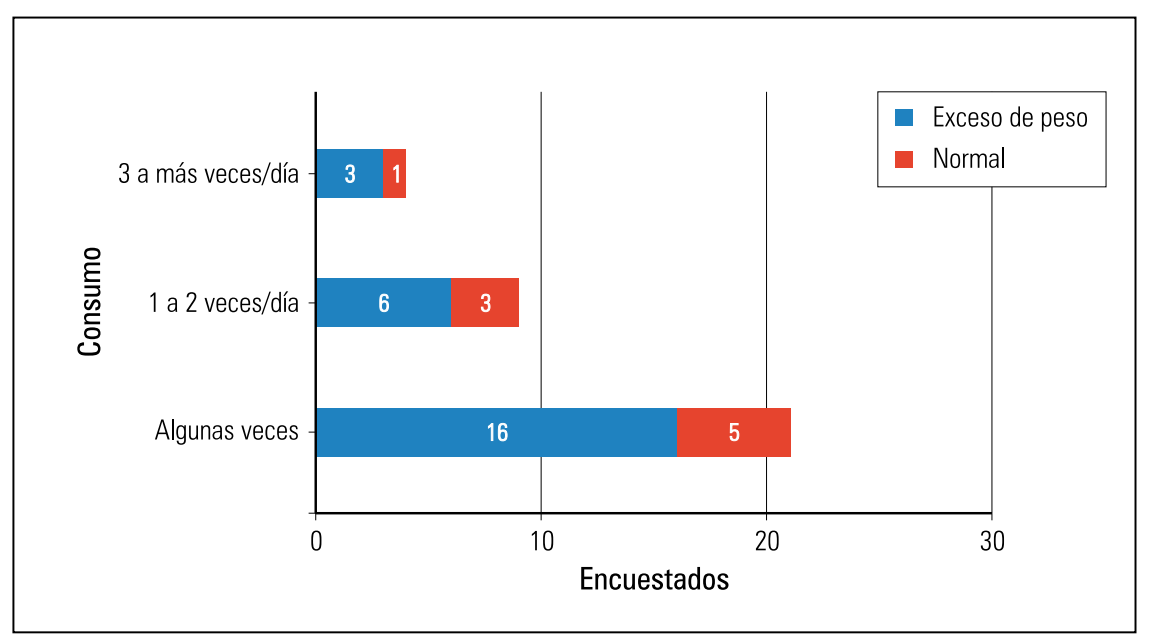

Figura 1. Distribución del consumo de frutas según el estado nutricional.

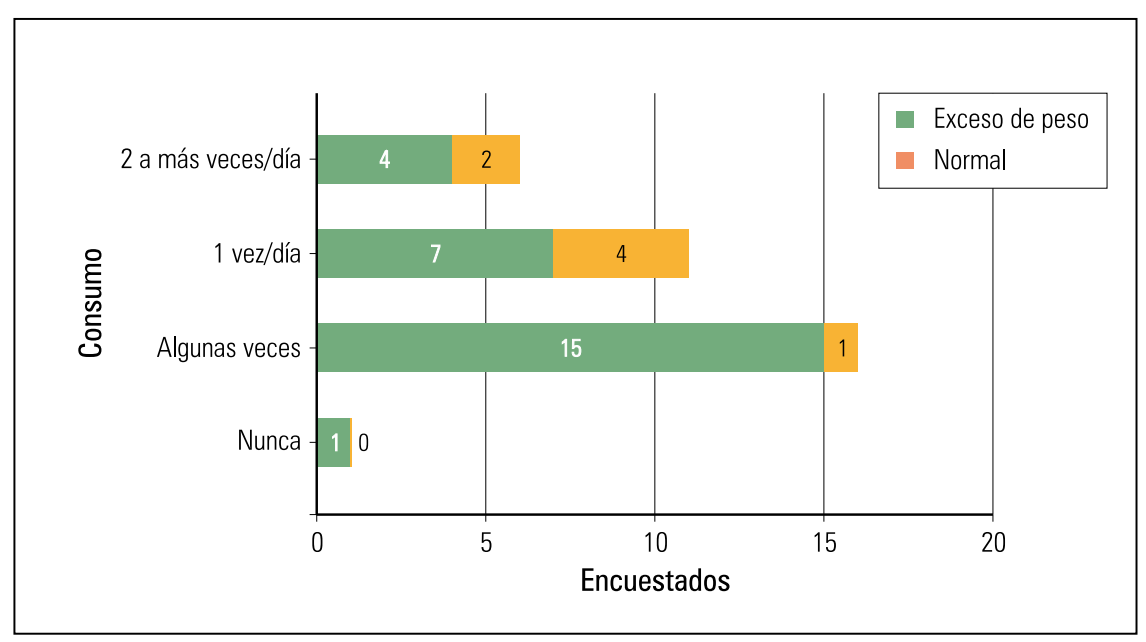

Figura 2. Distribución del consumo de verduras según el estado nutricional. mana). El promedio para los 3 tiempos de comida principales (desayuno, almuerzo, cena) en casa fue 4 veces a la semana.

El tiempo promedio dedicado a las principales comidas fue 9 minutos para el desayuno, 26 para el almuerzo y 27 para la cena.
Se encontró que el consumo de frutas fue esporádico, $62 \%$ consumía algunas veces fruta y tan solo $12 \%$ tenía un consumo apropiado de 3 veces al día o más (figura 1). Caso similar sucedió con el consumo de verduras (figura 2), encontrando que $47 \%$ de la muestra consumía verduras algunas veces, 29\%
Tabla 2. Consumo de los diferentes tiempos de comida, en casa.

\begin{tabular}{|c|c|c|c|}
\cline { 2 - 3 } & \multicolumn{2}{c}{ Come en casa } & Promedio de veces \\
Sín & No & 4 \\
\hline Desayuno & 29 & 5 & 3 \\
\hline Almuerzo & 27 & 7 & 5 \\
\hline Cena & 28 & 6 & \\
\hline
\end{tabular}




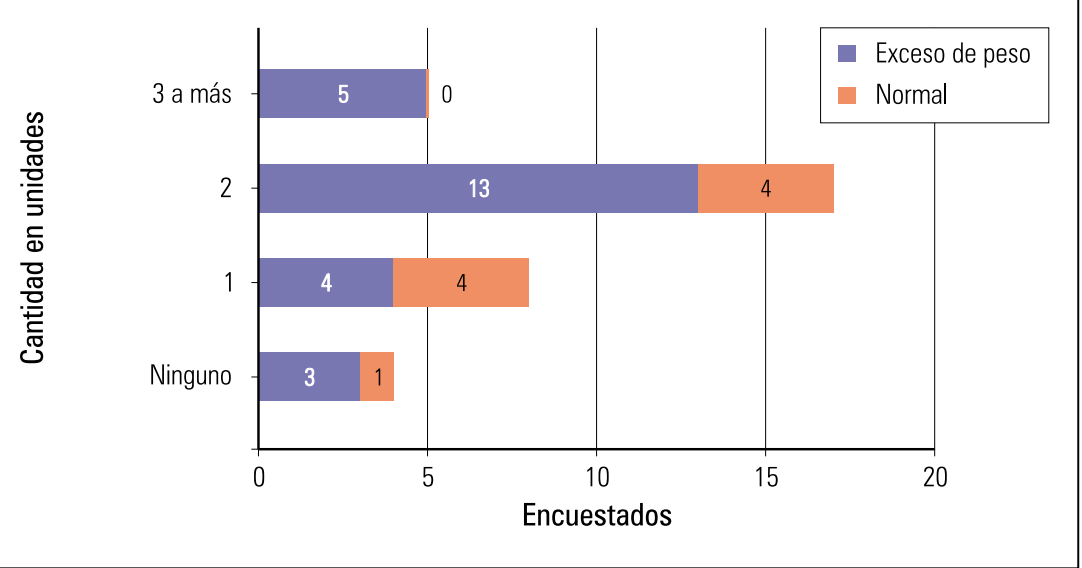

Figura 3. Consumo de pan según estado nutricional.

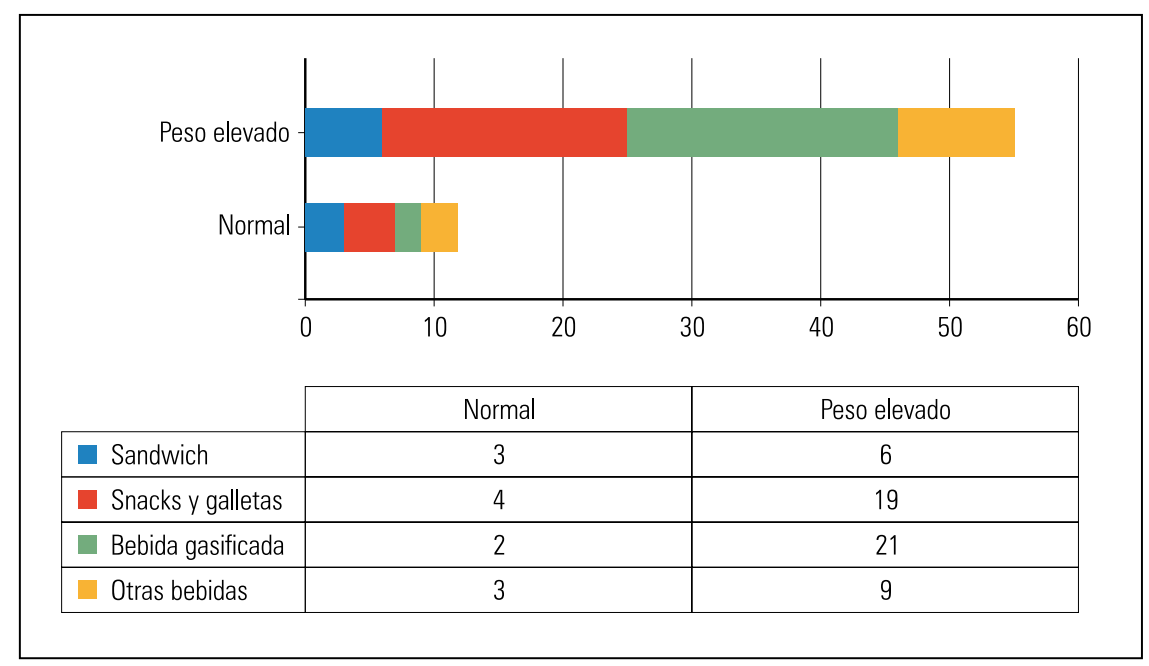

Figura 4. Preferencia de alimentos consumidos entre comidas, según el estado nutricional.

una vez al día y $18 \%$ dos veces al día o más, haciéndose evidente el consumo bajo de estos dos grupos de alimentos.

El consumo de pan fue medido por unidades al día (figura 3). Se encontró que $50 \%$ consumía dos panes por día, incluyendo la mayor cantidad de médicos residentes con exceso de peso. Sin embargo, el consumo de más de 3 panes diarios fue exclusivo de las personas con exceso de peso.

Del consumo de alimentos entre comidas, $71 \%$ lo hacía frecuentemen- te; de ellos, 18 (75\%) tenían exceso de peso y $6(25 \%)$, un estado nutricional normal.

La preferencia de alimentos consumida entre comidas fue clasificada en cuatro categorías. Al analizar la preferencia según el estado nutricional (figura 4), se encontró que las personas que tenían un peso elevado mostraban mayor preferencia por las bebidas gasificadas (38\%), mientras que las personas con estado nutricional normal tenían preferencia por los snacks y las galletas (33\%).
La preferencia por el consumo bajo de comidas condimentadas fue frecuente tanto para las personas con peso normal como en aquellas con peso elevado (figura 5).

La ingesta de comida dos horas antes de ir a dormir fue mayor en las personas con peso elevado (65\%) que en las personas con peso normal (35\%).

De la actividad que realizaban los médicos, 31 (91\%) de ellos continuaban laborando en el hospital luego de su guardia nocturna, solo 2 (6\%) tomaban un descanso de por lo menos 6 horas después de su guardia y uno $(6 \%)$ hacía actividades recreativas.

Sobre los hábitos nocivos, 53\% de los médicos residentes no tenía el hábito de fumar, de los cuales $72 \%$ presentaba peso elevado. Mientras que, para la ingesta de bebidas alcohólicas, 59\% lo hacía esporádicamente y de ellas $77 \%$ tenía peso elevado.

El 74\% de los médicos residentes no realizaba actividad física programada, constituyendo el grupo de personas con exceso de peso $72 \%$ de quienes no practicaban actividad física.

Del total de personas que practicaba una actividad física programada, $78 \%$ tenía peso elevado; 6 (60\%) practicaban una actividad física aeróbica y tenía peso elevado. Sin embargo, la frecuencia de dedicación a la actividad física era menor de 3 veces a la semana en $6(46 \%)$ residentes, resaltando que $6(86 \%)$ de los individuos que practicaban actividad física más de 3 veces a la semana también presentaban peso elevado. Doce $(92 \%)$ dedicaban más de 30 minutos a la práctica de deporte, siendo el grupo de peso elevado el más representativo, con 9 residentes (75\%).

Respecto a la relación que tenía el estado nutricional con la cercanía o convivencia con un familiar que tuviera peso elevado, 16 (63\%) de las personas con exceso de peso no convivía con una persona que tuviera la misma condición. 
En el caso de la percepción del estrés, 7 (90\%) de los individuos con peso normal y $15(60 \%)$ con peso elevado percibían que estaban frecuentemente estresados.

\section{DISCUSIÓN}

Los resultados de este estudio muestran una prevalencia de sobrepeso y obesidad ( 53 y $21 \%$, respectivamente) en los médicos residentes evaluados, dato de obesidad similar a la encontrada en la población general por el Ministerio de Salud en el año 2008, en la ciudad de Lima, de 22,8\% (5). Sin embargo, Palacios, en un estudio en trabajadores de la salud en México, en el año 2006, encontró una frecuencia de sobrepeso de $15 \%$ y de obesidad de $31 \%$, datos diferentes a los encontrados en nuestro estudio ${ }^{(7)}$.

Los médicos estudiados, para el desayuno tomaban un tiempo de 9 minutos, para el almuerzo 26 minutos y para la cena 27 minutos, datos que contrastan con lo recomendado por diferentes profesionales de la salud, que sugieren que para el desayuno se emplee por lo menos de 15 a 20 minutos.

El consumo de frutas y verduras resultó ser esporádico, con consumo inferior a lo recomendado (frutas 3 veces al día y verduras, 2 veces) y valores de $88 \%$ y $82 \%$ para frutas y verduras, respectivamente; solamente $12 \%$ y $18 \%$ se encontraban dentro de las recomendaciones para frutas como verduras. Dicho hallazgo resulta similar a lo encontrado por Castañola y col., en Buenos Aires, en una población de adolescentes: $86 \%$ no consumía frutas ni verduras durante el día, alrededor de $28 \%$ solo una o dos porciones/día y $1 \%$, cinco o más porciones/día; los autores determinaron que es un mal hábito alimentario que puede conllevar a la adquisición de enfermedades crónicas no transmisibles ${ }^{(8)}$.

La ingesta diaria de pan en los médicos residentes estuvo dentro de los parámetros normales o inferiores; sin embargo, de quienes consumían dos

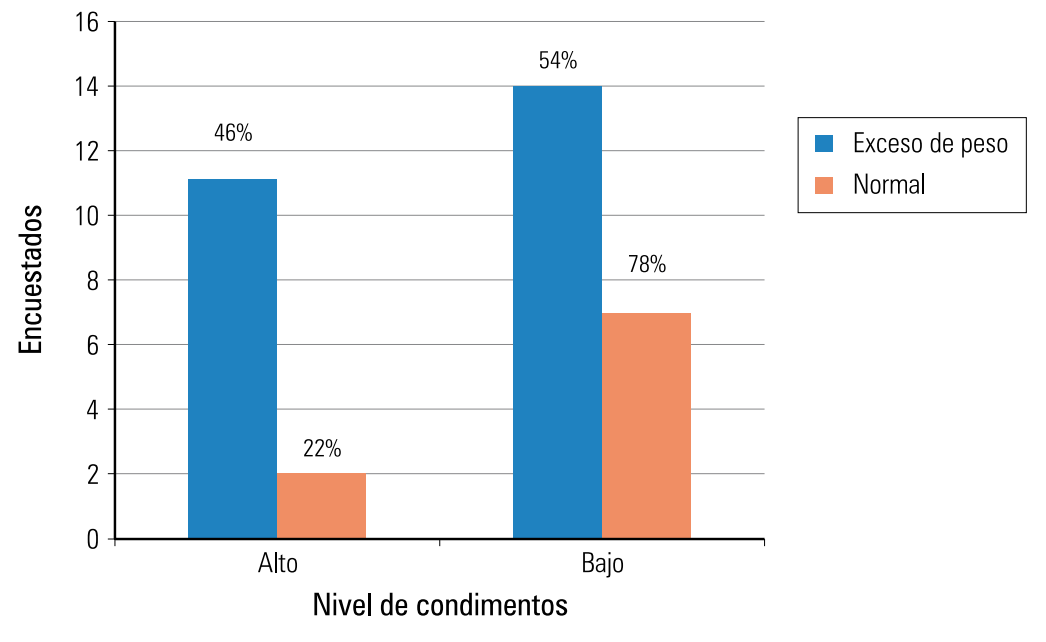

Figura 5. Preferencia por condimentación de la comida, según el estado nutricional.

unidades al día, $76 \%$ presentaba exceso de peso.

La ingesta de alimentos entre comidas fue frecuente en $71 \%$ de la población en estudio. Esto parece relacionarse con el estudio de Villarreal, en el año 2003, en la ciudad de San José, Costa Rica, que encontró que más de $50 \%$ de la población comía entre comidas ${ }^{(9)}$.

En nuestro estudio se halló que la preferencia de los alimentos ingeridos entre comidas era similar para ambos grupos. Sin embargo, la preferencia por los snacks, galletas y bebidas gasificadas fue más alta en la población con exceso de peso, siendo similar a lo escrito por Doval, en Argentina, el año 2008, quien describe una relación grande entre el consumo de comidas 'chatarra' con sobrepeso u obesidad y otras enfermedades consecuentes a estas ${ }^{(10)}$.

Según los datos obtenidos, $74 \%$ de los médicos no realizaba actividad física programada, considerándoseles sedentarios; representaron un nivel elevado en comparación con los datos publicados por el Ministerio de Salud, que comunicó que casi la mitad de los peruanos (48\%) era totalmente sedentaria, es decir, no realiza actividad física y se asocia directamente con el sobrepeso u obesidad ${ }^{(11)}$.
Se considera este nivel alto de sedentarismo por el tipo de ocupación laboral y las pocas horas dedicadas al autocuidado y recreación; en nuestros datos, $91 \%$ de los médicos continuaba trabajando y solo $6 \%$ tomaba un descanso de por lo menos 6 horas después de sus guardias nocturnas, lo cual está muy relacionado con la presencia de estrés ${ }^{(3)}$.

De quienes presentaban exceso de peso, $60 \%$ comunicó estrés, lo cual se relaciona con el elevado número de médicos (91\%) que continúa trabajando posteriormente a sus guardias nocturnas; Villarreal, en 1999, refirió que el exceso de trabajo y la mala alimentación conllevan al estrés ${ }^{(3)}$.

Terminamos alertando que no encontramos correlación significativa entre el estilo de vida y la prevalencia de sobrepeso u obesidad. Sin embargo, los hallazgos se semejan con los de la literatura relacionada al tema. También, hay que destacar que en muy pocas ocasiones se ha evaluado estos aspectos en el personal médico y menos aún con muestras representativas. Resaltamos la dificultad práctica que supone evaluar al personal médico ${ }^{(12)}$, esto dado por la negatividad de ser partícipe del estudio, así como por la escasa disponibilidad 
de tiempo para recabar la información necesaria. Aún así, hay que animar la realización de estudios que incluyan muestras representativas y optimizar las herramientas a utilizar, para así obtener datos más representativos y que permitan estudiar no solo los estilos de vida sino los hábitos alimentarios y el contenido de las dietas. De esta manera podría contrastarse si es que la dieta de los médicos influye directamente sobre la prevalencia elevada del sobrepeso u obesidad o si son otros los factores que desencadenan esta situación.

Se recomienda que en las instituciones hospitalarias se instaure un sistema de promoción y control de la salud del personal médico y demás profesionales, con la finalidad de tener un mejor estado de salud.

Concluyendo, el personal de residentes médicos presentó exceso de peso en $74 \%$ de la muestra, determinándose que no existió correlación significativa que relacionara el estilo de vida y la prevalencia del exceso de peso.

\section{REFERENCIAS BIBLIOGRÁFICAS}

1. Cortez R. Salud, equidad y pobreza en el Perú: Teoria y nuevas tendencias. Centro de Investigación de la Universidad del Pacifico. 1 ra Ed. Lima. 2002.

2. Hernández S. Fisiopatologia de la obesidad. Gac Med Méx. 2004;140(Supl 2):S31.

3. Arenas L, Hernández I, Valdez-Santiago R, Bonilla $P$. Las instituciones de salud y el autocuidado de los médicos. Salud Publ Mex. 2004;46(4):326-32.

4. Encuesta Nacional de Hogares sobre Medición de Niveles de Vida, 2000. Urbano - Rural. AID, BID, Telefónica del Perú, Terra Networks Perú S.A., GRADE, NEXTEL, Organización Panamericana de la Salud, Universidad del Pacífico, Instituto de Investigación Nutricional, The Brooking Institution. (Jun. 2000). http://www.cuanto.org/index. php? modulo $=56 \& a=3$

5. Ministerio de Salud. Encuesta nacional de indicadores nutricionales, bioquímicos, socio-económicos y culturales relacionados con las enfermedades crónicas no transmisibles. 2005. [base de datos en internet]. [citado 8 Mar 2009]. Disponible en: http://www.minsa.gob.pe/portal/Especiales/2007/ nutricion/publicaciones/INFORME_FINAL_ENIN. pdf.

6. Asociación Peruana de Obesidad y Ateroesclerosis. "La obesidad en el Perú". Lima Octubre 2008. [base de datos en internet]. [citado 20 Mar 2009]. Disponible en: http://www.ortomolecular-obesidad. com/2008/10/la-obesidad-en-el-per-ataca-al-26-de. html

7. Palacios-Rodriguez R, Munguia-Miranda C, ÁvilaLeyva A. Sobrepeso y obesidad en personal de salud de una unidad de medicina familiar. Rev Med Inst Mex Seguro Soc. 2006;44(5):449-53.
8. Castañola J, Magariños M, Ortiz S. Patrón de Ingesta de vegetales y frutas en adolescentes en el área metropolitana de Buenos Aires. Arch argent pediatr. 2004;102(4):265-70.

9. Villarreal S. Prevalencia de la obesidad, patologias crónicas no transmisibles asociadas y su relación con el estrés, hábitos alimentarios y actividad física en los trabajadores del Hospital de la Anexión. Rev Cienc Adm Financ Segur Soc. 2003;11(1):83-96.

10. Doval H. La epidemia de obesidad: ¿resolución individual o social? Rev Arg Cardiol. 2006;74(4):341-8.

11. Ministerio de Salud. La mitad de peruanos no realiza actividad física y corre riesgo de padecer enfermedades mortales. Abril 2009. . [base de datos en internet]. [citado 8 mar 2009]. Disponible en: http:// www.minsa.gob.pe/ocom/prensa/notadeprensa. asp?np_codigo $=7184 \&$ mes $=4 \&$ anio $=2009$.

12. Sánchez-Carracedo D, Saldaña C. Evaluación de los hábitos alimentarios en adolescentes con diferentes indices de masa corporal. Psicothema. 1998;10(2):281-92.

Trabajo presentado a las IX Jornadas Científicas Sanfernandinas, XII Jornadas de Investigación en Salud, XIX Jornadas Sanfernandinas Estudiantiles, Facultad de Medicina, UNMSM, setiembre 2010.

Correspondencia: Ivonne Bernui Leo

Correo electrónico: ibernuil@unmsm.edu.pe. 